\title{
Identifying Inaccessible Areas with Potential to Enhance Transit Market
}

\author{
Srinivas S. Pulugurtha, Venkata Ramana Duddu, Rakesh Mora \\ The University of North Carolina at Charlotte
}

\begin{abstract}
The focus of this manuscript is 1) to compute a transit accessibility index based on demographic/socio-economic characteristics and land use characteristics for each transit stop and route and the entire study area, and 2) identify inaccessible areas to provide an improved public transportation system that maximizes market potential. Transit accessibility indices were computed using spatially-extracted data within a pre-defined walking distance or time ( 0.25 miles or 5 minutes) for each transit stop (bus-stop) and route, and the entire study area. Results from linear regression analysis showed a statistically significant relationship between boardings and alightings, and the computed transit accessibility index based on demographic/socio-economic characteristics and land use characteristics for transit stops. The spatial distributions of computed transit accessibility indices were used to illustrate identification of spatial gaps, selection of ideal locations for transit stops along a route, extension of an existing route, identification of new transit routes, and expansion of transit area coverage.
\end{abstract}

\section{Introduction}

Rapid growth in population and travel demand over the past two decades has led to an increase in road congestion. This has been a major concern for not only transportation system managers but also to the traveling public. Literature documents several solutions to reduce huge economic and environmental losses associated 
with congestion. Providing an efficient, accessible, and affordable public transportation system is one such solution.

Increasing traffic volumes, suitable land development strategies, growing oil prices, and air pollution concerns could further contribute to commuters choosing transit as an alternate mode of transportation. However, public transportation systems managers may choke on their success if these systems are not planned, designed, built, and maintained to maximize transit market potential and ridership.

The successful deployment of public transportation systems often depend on adequate funding, service frequency, total travel time, fare, accessibility to the system, security, comfort, and convenience of travel. Building a public transportation system that considers these characteristics allows for provision of an affordable and efficient alternate mode of transportation to the general public. Such a system will not only be sustainable but supports a vibrant economy.

Accessibility depends on demographic/socio-economic characteristics and land use characteristics within the vicinity of a transit stop (say, a bus-stop). This manuscript focuses on 1) estimating a transit accessibility index based on demographic/ socio-economic characteristics and land use characteristics, and 2) identifying inaccessible areas in transit accessibility in order to enhance its market potential. It proposes a Geographic Information System (GIS) based methodology to compute a transit accessibility index for each transit stop (bus-stop), route, and study area as a whole (transit network-level performance). In this manuscript, spatial gaps or inaccessible areas are defined as areas with potential for transit market but do not have a transit stop within an acceptable walking distance and are not being served currently.

The spatial distributions of computed transit accessibility indices for transit stops and routes provide valuable insights in order to identify spatial gaps, select ideal locations for transit stops along a route, extend an existing route, identify new transit routes, and expand transit area coverage. Public transportation system managers can use these outcomes to better plan and serve the population in areas without transit service. This helps maximize transit market potential and ridership.

The working of the proposed GIS-based methodology is illustrated using bus transit system data for the city of Charlotte, North Carolina. The results obtained are assessed by examining the statistical relationship between 1) average daily boardings and the computed transit accessibility index for transit stops and 2) average daily alightings and the computed transit accessibility index for transit stops. 
The city of Charlotte does not have an extensive rail transit system (only one light rail corridor connecting South Charlotte to downtown/uptown, referred to as the Lynx Blue Line; opened in November 2007) at the time of this research. Rail transit system/ridership, therefore, has not been considered or discussed in this paper.

\section{Literature Review}

Past research on transit planning and accessibility dealt with various ways to measure an accessibility index. Sanchez (1998) suggested that transit access is a significant factor for assessing the average rate of labor participation. Pulugurtha et al. (1999) defined a measure for accessibility to a transit service facility based on potential captive riders with certain demographic characteristics such as age group, household income, ethnicity, household auto ownership, unemployment, and persons with physical disabilities living in a household.

Bhat et al. (2002) developed an urban accessibility index based on factors (such as information available, hours of operation, travel time choice models, and opportunities that vary with distance) and found varying results depending on the type of measure used. Beimborn et al. (2003) used accessibility and connectivity to identify potential captive riders. Lee (2004) recommended a parcel level measure of public transit accessibility to destinations using GIS. This method considered walk time, waiting time, and travel time using transit service and by walking. Kuby et al. (2004) developed a raster-based algorithm for determining off-network routes to identify trip origins and destinations.

Kimpel et al. (2007) used GIS to measure the effect of overlapping service areas on passenger boarding at transit stops. A distance decay function was used to calculate walking accessibility from dwelling units to transit stops.

Spacing between transit stops is another criterion that affects accessibility and transit planning. Research by Ammons (2001) on transit stop spacing standards recommended that the range of spacing in urban areas should be from $656 \mathrm{ft}$ to $1,968 \mathrm{ft}$. Saka (2001) developed an optimization model to determine the optimum spacing between transit stops in urban areas. Murray (2003) developed a coverage model to improve public transit system accessibility by minimizing the number of transit stops (bus stops) and maximizing the proportion of population covered by the transit stops. 


\section{Limitations of Past Research}

Research in the past focused primarily on accessibility for either a single transit stop or facilities along a transit route. Very little has been done to compute transit accessibility and compare it for multiple routes. In addition, not many authors focused on network-level performance that allows decision makers to analyze and assess the overall performance of a transit system to maximize market potential.

Demographic/socio-economic characteristics and land use characteristics of an area can be used to compute transit trip-related productions (number of boardings) or attractions (number of alightings). The type of transit activity (boardings or alightings) at a transit stop varies by the time of the day. As an example, a transit stop in a residential area may generate boardings during the morning peak hour but may attract alightings during the evening peak hour. Likewise, a transit stop in downtown/uptown or an office area may attract alightings during the morning peak hour but may generate boardings during the evening peak hour. On the other hand, there may be a few transit stops with similar ridership patterns (alightings and boardings) during regular travel hours on a day (example, near shopping malls). Not many authors in the past considered factors related to productions and attractions while defining a transit accessibility index.

This manuscript illustrates the working of a GIS-based methodology to compute transit accessibility index based on demographic/socio-economic characteristics and land use characteristics for all transit stops and routes in the study area. It also discusses an accessibility index as an area-wide measure to indicate a transit system's performance at a network level. The computed transit accessibility indices help not only to identify new transit stops, new routes, or the need for extension of an existing route but also increases market potential by minimizing spatial gaps and inaccessible areas. The subject research effort is an extension to transit stop accessibility based on demographic data discussed in Pulugurtha et al. (2011).

\section{Methodology}

The proposed GIS-based methodology to compute a transit accessibility index comprises the following steps:

1. Select variables.

2. Conduct spatial analysis.

3. Process data. 
4. Compute accessibility index for each transit stop.

5. Compute accessibility index for each transit route

6. Compute accessibility index for entire study area.

Each of the above steps is discussed next in detail.

\section{Selection of Variables}

The focus of this step is to select variables to compute the transit accessibility index based on demographic/socio-economic characteristics and land use characteristics that typically are used to estimate trip productions and attractions in a traditional four-step planning process. The variables to compute the transit accessibility index may vary from one area to another area. The thresholds or criterion (such as age group and income level) may also vary from one to another area.

As an example, the demographic/socio-economic characteristics to compute bus transit accessibility for a city of size and characteristics similar to city of Charlotte may include the unemployed, the population that belongs to households with 0 or 1 automobiles, the population by age group ( $15-64)$, and low income population $(<\$ 25,000)$.

The land use characteristics to compute the transit accessibility index for a city of size and characteristics similar to Charlotte may include residential, heavy/light commercial, heavy/light industrial, and institutional (comprising major educational, medical, government, cultural and religious, and other institutions) areas within the accessible area.

Transit riders can be categorized into captive riders and preferred riders. Captive riders are potential riders who do not have a choice other than to use transit system for travel to their destination. Preferred riders, on the other hand, are transit riders by choice, irrespective of their socio-economic status (such as household income). Variables pertaining to demographic/socio-economic characteristics and land use characteristics that are selected to compute the transit accessibility index should, therefore, account for both the categories of riders.

\section{Spatial Analysis}

Spatial analysis is carried out to compute the transit accessibility index based on demographic/socio-economic characteristics and land use characteristics. In this research, transit accessibility is defined in terms of walking distance or time for a rider to/from a transit stop from/to an origin/destination. Buffers representing this pre-defined walking distance ( 0.25 miles) or walking time ( 5 minutes) are generated 
around each transit stop/route in the transit system. A 0.25 mile buffer was considered as anecdotal evidence indicates that this is an acceptable walking distance to access bus-stops considered in this research. A larger (or different) buffer width needs to be considered if acceptable walking distance is longer/shorter or when analyzing other forms of transit systems such as commuter rail.

Figure 1 shows an example 0.25 mile buffer around a bus-stop. The data layers with demographic/socio-economic characteristics and land use characteristics are then overlaid on the generated buffers to extract/capture data pertaining to variables that help compute the transit accessibility index.

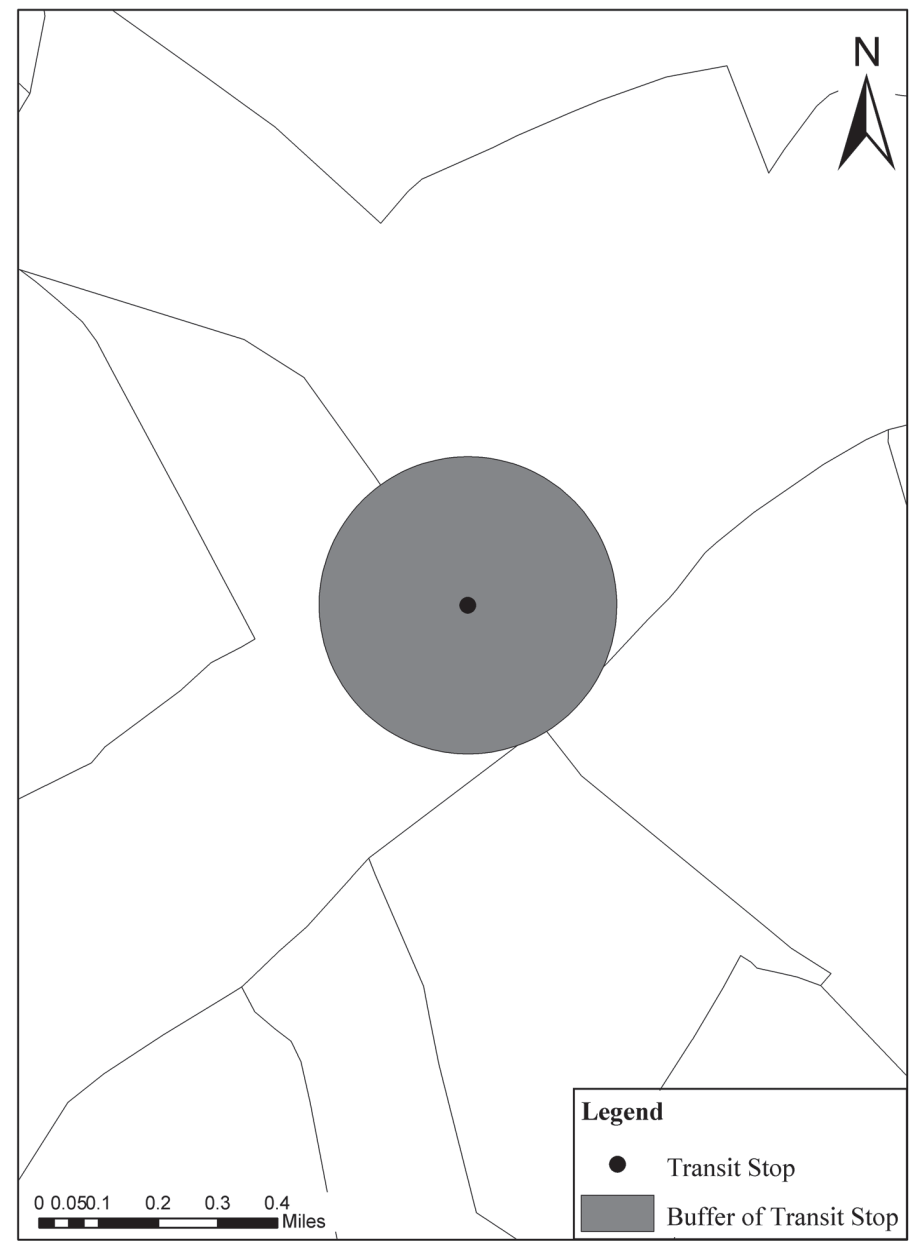

Figure 1. Buffer around a transit stop. 


\section{Data Processing}

The spatial overlay of data on generated buffers does not automatically adjust/recalculate the data attributes based on the area that falls within a buffer around a transit stop or a route. Data are therefore processed to compute values pertaining to each selected variable. As an example, the demographic/socio-economic data layer is overlaid on the generated buffers to compute the total number of unemployed in each buffer around each transit stop. The total number of unemployed in a buffer around a transit stop is then computed using the following equation.

$$
U_{i}=\sum_{j} \frac{A_{j, i}}{A_{j}} * U_{j}
$$

where,

$\mathrm{U}_{\mathrm{i}}=$ total number of unemployed in the buffer " $\mathrm{i}$ " around transit stop " $\mathrm{s}$ "

$\mathrm{U}_{\mathrm{j}}=$ total number of unemployed in census block "j"

$$
\begin{aligned}
& A_{j, i}=\text { area of census block " } j \text { " in buffer "i" around transit stop "s" } \\
& A_{j}=\text { area of census block " } j \text { " }
\end{aligned}
$$

Similarly, equations are developed to extract the population that belongs to households with 0 or 1 automobile, population by age group, and low income population in each generated buffer.

To extract office and commercial type land use characteristics, the land use data layer is overlaid on the generated buffers to compute the area of each land use characteristic within each buffer.

\section{Compute Accessibility Index for Each Transit Stop}

The extracted demographic/socio-economic characteristics and land use characteristics around each transit stop are normalized with respect to the value of the same characteristic to compute the transit accessibility index for a transit stop. As an example, the normalized score for the number of unemployed in the buffer " $i$ " around transit stop " $s$ " is computed as shown in the Equation (2). 
where,

$$
\mathrm{U}_{\mathrm{s}}=\frac{\mathrm{U}_{\mathrm{i}}}{\operatorname{Maximum}\left(\mathrm{U}_{\mathrm{i}}\right)}
$$

$\mathrm{U}_{\mathrm{s}} \quad=$ normalized score for the total unemployed in the buffer around transit stop "s",

$U_{i}=$ total number of unemployed in the buffer " $\mathrm{i}$ " around transit stop " $\mathrm{s}$ ", and,

Maximum $\left(U_{i}\right)=$ maximum number of unemployed considering all the buffers around transit stops.

The accessibility index for each transit stop "s" based on demographic/socio-economic data are computed using the following equation.

$$
\mathrm{A}_{\mathrm{d}, \mathrm{s}}=\mathrm{U}_{\mathrm{s}}+\mathrm{AO}_{\mathrm{s}}+\mathrm{AG}_{\mathrm{s}}+\mathrm{I}_{\mathrm{s}}
$$

where,

$\mathrm{A}_{\mathrm{d}, \mathrm{s}}=$ transit accessibility index based on demographic/socio-economic characteristics for transit stop "s"

$\mathrm{U}_{\mathrm{s}} \quad=$ normalized score for the total number of unemployed in the buffer for transit stop "s"

$\mathrm{AO}_{\mathrm{s}}=$ normalized score for population with auto-ownership 0 or 1 in the buffer for transit stop "s"

$\mathrm{AG}_{\mathrm{s}}=$ normalized score for population with age group between 15 to 64 in the buffer for transit stop "s"

$\mathrm{I}_{\mathrm{s}}=$ normalized score for low income population in the buffer for transit stop "s"

Similarly, the accessibility index based on land use characteristics for transit stop "s" is computed using following equation.

$$
\mathrm{A}_{1, \mathrm{~s}}=\mathrm{HC}_{\mathrm{s}}+\mathrm{LC}_{\mathrm{s}}+\mathrm{HI}_{\mathrm{s}}+\mathrm{LI}_{\mathrm{s}}+\mathrm{I}_{\mathrm{s}}
$$

where, 
$\mathrm{A}_{\mathrm{l}, \mathrm{s}}=$ transit accessibility index based on land use characteristics for transit stop "s"

$\mathrm{HC}_{\mathrm{s}}=$ normalized score for total heavy commercial land use area in the buffer for transit stop "s"

$\mathrm{LC}_{\mathrm{s}}=$ normalized score for total light commercial land use area in the buffer for transit stop "s"

$\mathrm{HI}_{\mathrm{s}}=$ normalized score for total heavy industrial land use area in the buffer for transit stop " $\mathrm{s}$ "

$\mathrm{LI}_{\mathrm{s}} \quad$ = normalized score for total light industrial land use area in the buffer for transit stop " $\mathrm{s}$ "

$\mathrm{I}_{\mathrm{s}}=$ normalized score for total institutional land use area in the buffer for transit stop "s"

The computed transit accessibility indices in this step can be used to eliminate any transit stops with very low values for improving transit operations. This will help lower travel or run time, making the system more attractive. Eliminating stops with very few users also improves transit operational performance.

\section{Compute Accessibility Index for Each Route}

Buffers generated for all transit stops along a transit route are dissolved to compute transit accessibility index indicators for route "r." Demographic/socio-economic and land use data layers are then overlaid to extract data pertaining to various characteristics for measuring transit accessibility index indicators at the route level.

To assess route level accessibility, the total accessibility index based on dissolved buffers around transit stops is compared to the total transit accessibility index based on demographic/socio-economic data for the same route " $r$ " extracted by generating a buffer around the route.

The transit accessibility index based on demographic/socio-economic data for route " $r$ " is computed using the following equation.

$$
\mathrm{A}_{\mathrm{d}, \mathrm{r}, \mathrm{t}}=\mathrm{U}_{\mathrm{r}, \mathrm{t}}+\mathrm{AO}_{\mathrm{r}, \mathrm{t}}+\mathrm{AG}_{\mathrm{r}, \mathrm{t}}+\mathrm{I}_{\mathrm{r}, \mathrm{t}}
$$

where, 
$A_{d, r, t}=$ transit accessibility index based on demographic/socio-economic data for transit route " $r$ "

$\mathrm{t}=$ type (based on dissolved buffers around transit stops or a generated buffer along transit route " $r$ ")

$U_{r, t}=$ total number of unemployed in buffer type " $t$ " along transit route " $r$ "

$\mathrm{AO}_{\mathrm{r}, \mathrm{t}}=$ population with auto-ownership 0 or 1 in buffer type " $\mathrm{t}$ " along transit route " $r$ "

$A G_{r, t}=$ population with age group between 15 to 64 in buffer type " $t$ " along transit route " $r$ "

$I_{r, t}=$ low income population in buffer type " $t$ " along transit route " $r$ "

Apart from indicating the level of accessibility to a transit system along a route, spatial overlay of accessibility indicators based on demographic/socio-economic characteristics for route " $r$ " helps to identify spatial gaps or inaccessible areas along the route. The information can be used to add, remove or relocate existing transit stops so as to maximize ridership (market potential) along the route.

Similarly, the transit accessibility index based on land use characteristics for transit route " $r$ " using dissolved buffers around transit stops and buffer around the transit route are computed using Equation (6) and compared to identify spatial gaps or inaccessible areas along the route.

$$
\mathrm{A}_{\mathrm{l}, \mathrm{r}, \mathrm{t}}=\mathrm{HC}_{\mathrm{r}, \mathrm{t}}+\mathrm{LC}_{\mathrm{r}, \mathrm{t}}+\mathrm{HI}_{\mathrm{r}, \mathrm{t}}+\mathrm{LI}_{\mathrm{r}, \mathrm{t}}+\mathrm{I}_{\mathrm{r}, \mathrm{t}}
$$

where,

$\mathrm{A}_{\mathrm{l}, \mathrm{r}, \mathrm{t}}=$ transit accessibility index based on land use characteristics for transit route " $r$ "

$\mathrm{t} \quad=$ type (based on dissolved buffers around transit stops or a generated buffer along transit route " $r$ ")

$\mathrm{HC}_{\mathrm{r}, \mathrm{t}}=$ total heavy commercial land use area in buffer type " $\mathrm{t}$ " for transit route "r" 
$\mathrm{LC}_{\mathrm{r}, \mathrm{t}}=$ total light commercial land use area in buffer type " $\mathrm{t}$ " along transit route
" $\mathrm{r}$ "

$\mathrm{HI}_{r, \mathrm{t}}=$ total heavy industrial land use area in buffer type " $\mathrm{t}$ " along transit route " $r$ "

$\mathrm{LI}_{\mathrm{r}, \mathrm{t}}=$ total light industrial land use area in buffer type " $\mathrm{t}$ " along transit route " $r$ "

$\mathrm{I}_{\mathrm{r}, \mathrm{t}}=$ total institutional land use area in buffer type " $\mathrm{t}$ " along transit route " $\mathrm{r}$ "

\section{Compute Accessibility Index for Entire Study Area}

Buffers generated around each transit stop in the study area are dissolved to compute transit accessibility indicators for the entire study area (transit network level performance). Demographic/socio-economic and land use data layers are then overlaid to extract data pertaining to various characteristics for measuring transit accessibility index indicators at the network level.

To assess network level accessibility, the total accessibility index based on demographic/socio-economic data for the entire study area using dissolved buffers around transit stops is compared to the total accessibility index based on demographic/socio-economic data for the entire study area using census block level data.

The accessibility index based on demographic/socio-economic data for the entire study area using dissolved buffers or census block level data is computed using the following equation.

$$
\mathrm{A}_{\mathrm{d}, \mathrm{e}, \mathrm{o}}=\mathrm{U}_{\mathrm{e}, \mathrm{o}}+\mathrm{AO}_{\mathrm{e}, \mathrm{o}}+\mathrm{AG}_{\mathrm{e}, \mathrm{o}}+\mathrm{I}_{\mathrm{e}, \mathrm{o}}
$$

where,
$\mathrm{A}_{\mathrm{d}, \mathrm{e}, \mathrm{o}}=$ transit accessibility index based on demographic/socio-economic data using option "o" for the entire study area " $\mathrm{e}$ "
o = type (first one is based on dissolved buffers around transit stops while second one is based on census block level data)
$U_{e, 0}=$ total number of unemployed using option " $\mathrm{o}$ " for the entire study area "e" 
$\mathrm{AO}_{\mathrm{e}, \mathrm{o}}=$ population with auto-ownership 0 or 1 using option "o" for the entire study area "e"

$A G_{e, 0}=$ population with age group between 15 to 64 using option "o" for the entire study area "e"

$\mathrm{I}_{\mathrm{e}, \mathrm{o}} \quad$ = low income population using option "o" for the entire study area " $\mathrm{e}$ "

The spatial overlay of transit accessibility indicators based on demographic/socioeconomic data by transit stop on the map based on census block level data and street network helps identify possible new transit routes or possible extensions of existing routes for improved coverage.

Similarly, the transit accessibility index based on land use characteristics for the entire study area based on dissolved buffers around transit stops are computed using Equation (8) and compared to identify spatial gaps or inaccessible areas along the route.

$$
\mathrm{A}_{\mathrm{l}, \mathrm{e}, \mathrm{o}}=\mathrm{HC}_{\mathrm{e}, \mathrm{o}}+\mathrm{LC}_{\mathrm{e}, \mathrm{o}}+\mathrm{HI}_{\mathrm{e}, \mathrm{o}}+\mathrm{LI}_{\mathrm{e}, \mathrm{o}}+\mathrm{I}_{\mathrm{e}, \mathrm{o}}
$$

where,

$\mathrm{A}_{\mathrm{l}, \mathrm{e}, \mathrm{O}}=$ transit accessibility index based on land use characteristics using option "o" for the entire study area " $\mathrm{e}$ "

o = option (based on dissolved buffers around transit stops or study area level land use data)

$\mathrm{HC}_{\mathrm{e}, \mathrm{o}}=$ total heavy commercial land use area using option "o" for the entire study area "e"

$\mathrm{LC}_{\mathrm{e}, \mathrm{o}}=$ total light commercial land use area using option " $\mathrm{o}$ " for the entire study area "e"

$\mathrm{H}_{\mathrm{e}, \mathrm{o}}$ = total heavy industrial land use area using option "o" for the entire study area "e"

$\mathrm{LI}_{\mathrm{e}, \mathrm{o}}$ = total light industrial land use area using option "o" for the study area "e"

$\mathrm{I}_{\mathrm{e}, \mathrm{o}} \quad=$ total institutional land use area using option "o" for the entire study area "e" 


\section{Analysis and Results}

Bus transit network data for the city of Charlotte are used to illustrate the working of the methodology. The data obtained and used in the analysis includes 2008 bus transit network and ridership data from Charlotte Area Transit System (CATS), 2008 land use data from the Charlotte Department of Transportation, and the census block level data (2008).

The bus transit system in the city of Charlotte is operated by CATS. There are 80 transit routes and 3,645 bus stops in the study area. As stated previously, the existing light rail corridor (referred to as the Lynx Blue Line) was not considered, as the emphasis of this research is more on a bus-operated transit system.

Demographic/socio-economic and land use characteristics were extracted for all the 3,645 bus-stops in the study area. Data extracted include the unemployed, the population that belongs to households with 0 or 1 automobiles, the population by age group $(15-64)$, low income population $(<\$ 25,000)$, heavy/light commercial area, heavy/light industrial area, and institutional area.

Findings from a report published by Acs and Loprest (2005) based on National Survey of American Families was used to establish the criterion for low income population. Residential land use characteristics were not considered as they were found to have a strong correlation with demographic/socio-economic characteristics. Anecdotal evidence as well as discussions with staff of local agencies indicates that these are reasonable assumptions for the study area considered for illustration of the GIS-based methodology discussed in this manuscript.

Transit accessibility indices were then computed for each transit stop and route and the entire study area.

\section{Transit Accessibility Index for Transit Stops}

Figure 2 depicts the transit accessibility index based on demographic/socio-economic data by transit stop in the study area. The size of the point representing each transit stop is defined as a function of its transit accessibility index - the higher the value of the transit accessibility index, the larger the size of the point. The average transit accessibility index value based on demographic/socio-economic data is 0.72 , whereas the maximum value is 3.28 . Similarly, the average and maximum transit accessibility index based on land use characteristics are 0.30 and 1.10 , respectively. 


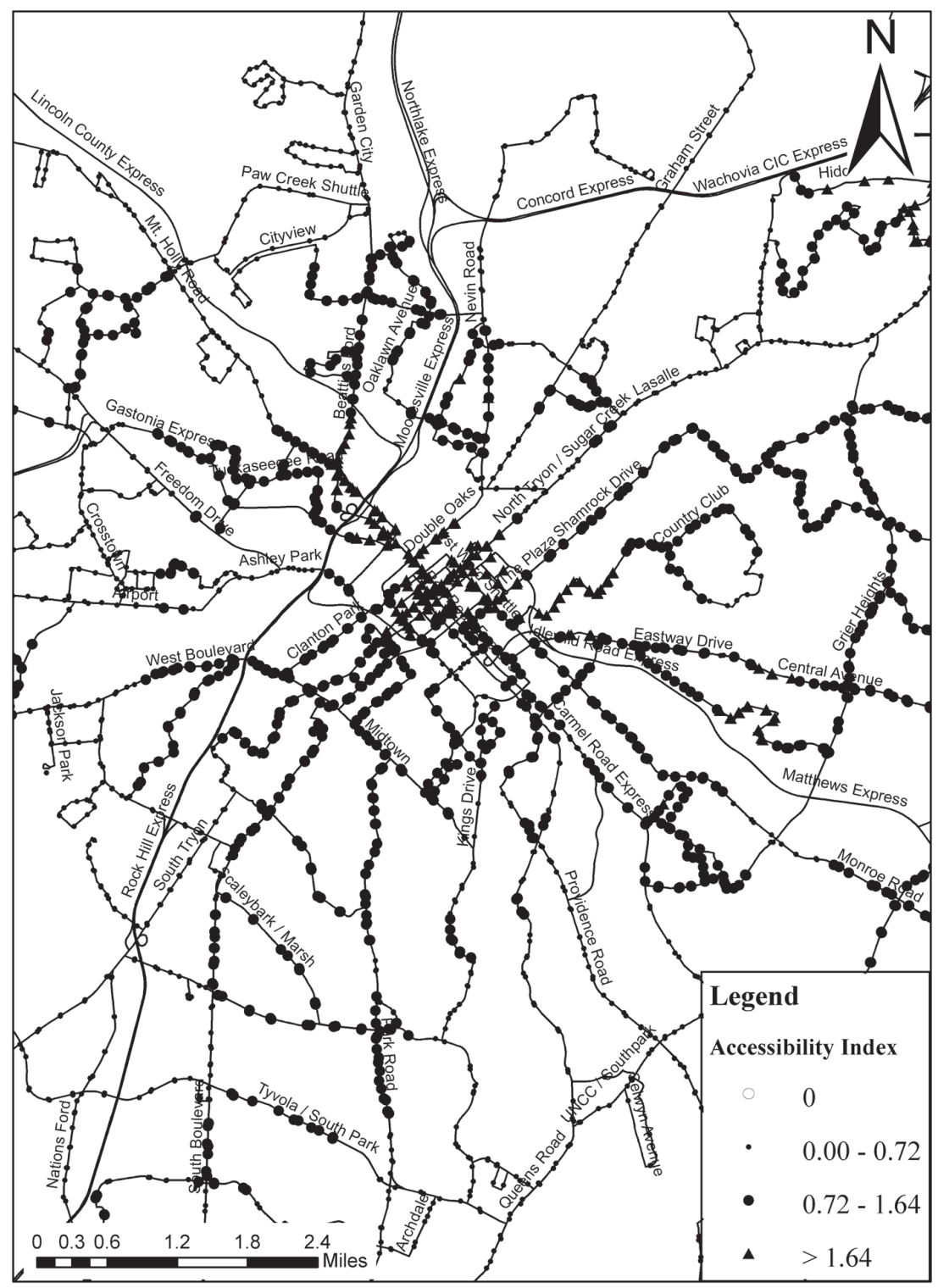

Figure 2. Spatial distribution of accessibility index based on demographic/socio-economic data for transit stops. 
Table 1 summarizes both the computed transit accessibility indices by range for all the transit stops. The transit accessibility ranges in the table are divided based on average and standard deviation values. Results shown in Table 1 indicate that 57.07 percent and 58.65 percent of transit stops have transit accessibility index values less than the average value of the transit accessibility index based on demographic/ socio-economic data and land use characteristics, respectively. On the other hand, 6.06 percent and 5.02 percent of transit stops have transit accessibility index values greater than the average plus two standard deviations based on demographic/ socio-economic and land use characteristics, respectively.

Table 1. Summary of Accessibility Index by Transit Stops

\begin{tabular}{|c|c|c|}
\hline Transit Accessibility Index Range & \# Transit Stops & \% Transit Stops \\
\hline \multicolumn{3}{|c|}{ Demographic/Socio-economic } \\
\hline 0 & 0 & 0.00 \\
\hline 0.01 to 0.72 & 2,090 & 57.07 \\
\hline 0.72 to 1.64 & 1,333 & 36.40 \\
\hline$>1.64$ & 222 & 6.06 \\
\hline \multicolumn{2}{|c|}{ Land Use } \\
\hline 0 & 470 & 12.83 \\
\hline 0.01 to 0.30 & 1,678 & 45.82 \\
\hline 0.30 to 0.86 & 1,313 & 35.85 \\
\hline$>0.86$ & 184 & 5.02 \\
\hline
\end{tabular}

The spatial overlay of transit accessibility indices and information from Table 1 (with more breakdown in range) assists in the decision making process. As an example, one can remove transit stops with 0 transit accessibility index based on land use characteristics and very low transit accessibility index based on demographic/socio-economic characteristics to improve transit operation or service along a transit route (say, reduce travel or run time).

\section{Transit Accessibility Index by Route}

Transit accessibility indices are computed for each route in the study area using the proposed methodology. As an example, Table 2 summarizes the computed accessibility indicators based on demographic/socio-economic characteristics and land use characteristics along Route 2 in the study area. 
Table 2. Route Level Analysis Summary - Example Route 2

(a) Demographic/Socio-economic

\begin{tabular}{|l|c|c|c|c|c|}
\hline Category & $\mathbf{U}_{\mathbf{r}, \mathbf{t}}$ & $\mathbf{A O}_{\mathbf{r}, \mathbf{t}}$ & $\mathbf{A G}_{\mathrm{r}, \mathrm{t}}$ & $\mathbf{I}_{\mathbf{r}, \mathbf{t}}$ & $\mathbf{A}_{\mathrm{d}, \mathrm{r}, \mathrm{t}}$ \\
\hline $\begin{array}{l}\text { Based on dissolved buffers along transit stops } \\
\text { of Route 2 }\end{array}$ & 487 & 1,959 & 4,103 & 968 & 7,517 \\
\hline Based on buffer generated along Route 2 & 558 & 2,349 & 5,119 & 1,168 & 9,194 \\
\hline \% Not Served & 12.64 & 16.61 & 19.85 & 17.14 & 18.24 \\
\hline
\end{tabular}

(b) Land Use

\begin{tabular}{|l|c|c|c|c|c|c|}
\hline Category & $\mathbf{H C}_{r, t}$ & $\mathbf{L C}_{r, t}$ & $\mathbf{H I}_{r, t}$ & $\mathbf{L I}_{r, t}$ & $\mathbf{I}_{r, t}$ & $\mathbf{A l}_{r, t}$ \\
\hline $\begin{array}{l}\text { Based on dissolved buffers along transit stops } \\
\text { of Route 2 }\end{array}$ & 0.56 & 0.14 & 0.15 & 0.26 & 0.09 & 1.21 \\
\hline Based on buffer generated along Route 2 & 0.69 & 0.33 & 0.40 & 0.43 & 0.10 & 1.95 \\
\hline \% not served & 18.69 & 56.64 & 62.57 & 38.85 & 4.37 & 37.93 \\
\hline
\end{tabular}

Along Route 2, 12.64 percent of unemployed, 16.61 percent of population with auto-ownership 0 or $1,19.85$ percent of population between 15 to 64 years of age, and 17.14 percent of population with income less than $\$ 25,000$ are not being served along this route. The overall transit accessibility index based on existing transit stops along Route $2(7,517)$ is 81.76 percent of the maximum potential transit accessibility index based on criteria used in this manuscript $(9,194)$.

The results obtained indicate that 18.69 percent, 56.64 percent, 62.57 percent, 38.85 percent, and 4.37 percent of heavy commercial, light commercial, heavy industrial, light industrial, and institutional land use areas, respectively, are not being served currently along Route 2 . Overall, 37.93 percent of these land use types are not being served by transit stops along the route.

Figure 3 shows the dissolved buffer along transit stops and the buffer generated along Route 2. The figure can be used to identify spatial gaps (inaccessible areas) based on demographic/socio-economic and land use characteristics along the route. Possible addition of new transit stops (based on optimal stop-spacing) in these gaps along the transit route could potentially increase transit market potential. 


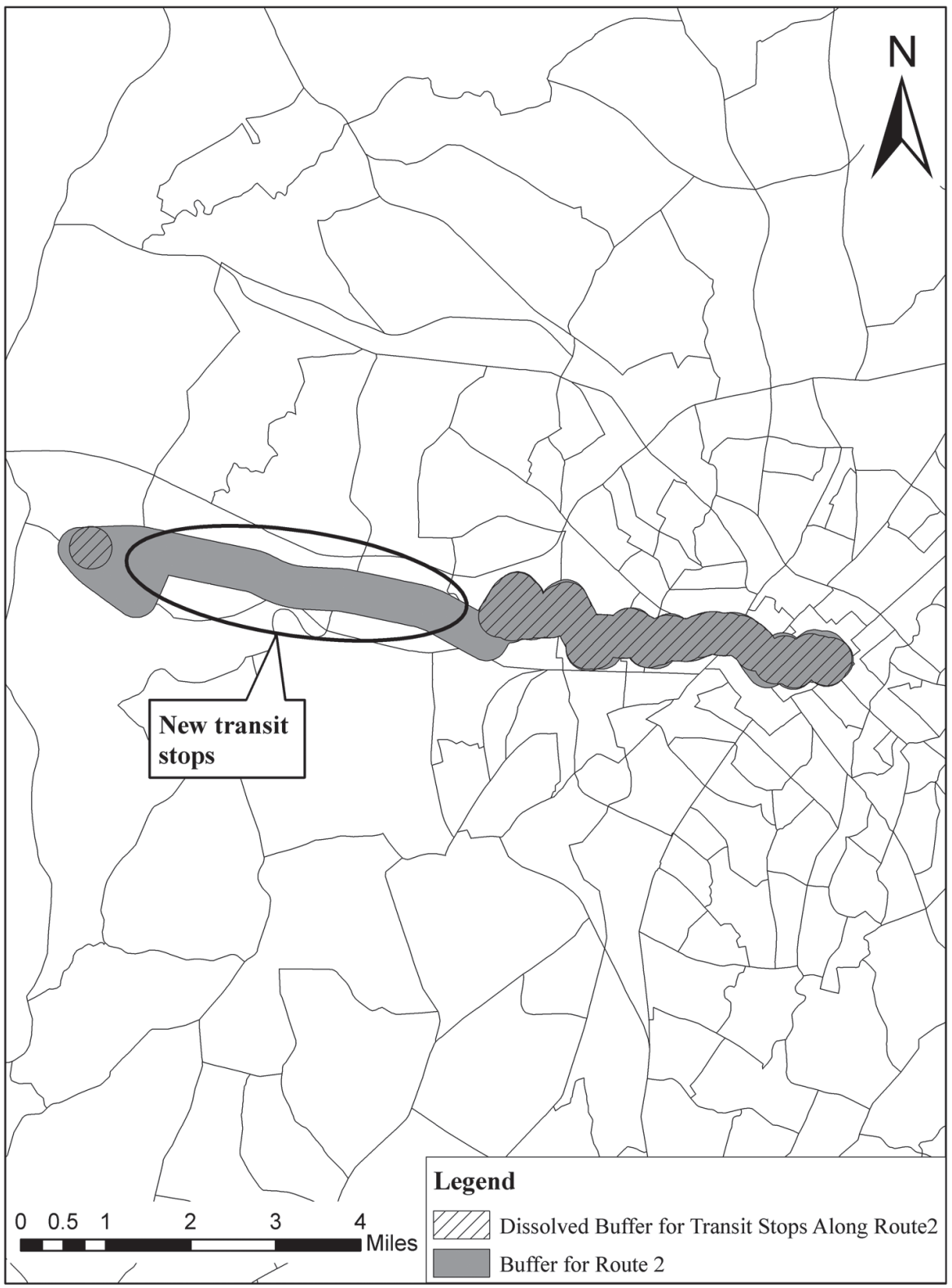

Figure 3. Identifying spatial gaps along Route 2. 


\section{Area-wide Accessibility Index}

Table 3 summarizes the computed transit accessibility indicators based on demographic/socio-economic characteristics and land use characteristics for the entire study area.

\section{Table 3. Network Level Analysis Summary}

(a) Demographic/Socio-economic

\begin{tabular}{|l|l|l|l|l|l|}
\hline Category & $\mathbf{U}_{\mathbf{e}, \mathbf{o}}$ & $\mathbf{A O}_{\mathrm{e}, \mathbf{o}}$ & $\mathbf{A G}_{\mathrm{e}, \mathrm{o}}$ & $\mathbf{I}_{\mathbf{e}, \mathbf{o}}$ & $\mathbf{A d}_{\mathbf{e}, \mathbf{o}}$ \\
\hline $\begin{array}{l}\text { Based on dissolved buffers along } \\
\text { transit stops in the study area }\end{array}$ & 18,686 & 79,442 & 248,053 & 30,369 & 376,550 \\
\hline $\begin{array}{l}\text { Based on study area census block level } \\
\text { data }\end{array}$ & 36,197 & 150,979 & 604,556 & 50,888 & 842,620 \\
\hline$\%$ Not Served & 48.38 & 47.38 & 58.97 & 40.32 & 55.31 \\
\hline
\end{tabular}

(b) Land Use

\begin{tabular}{|l|l|l|l|l|l|l|}
\hline Category & $\mathbf{H C}_{\mathbf{e}, \mathbf{o}}$ & $\mathbf{L C}_{\mathbf{e}, \mathbf{o}}$ & $\mathbf{H I}_{\mathbf{e}, \mathbf{o}}$ & $\mathbf{L l}_{\mathbf{e}, \mathbf{o}}$ & $\mathbf{I}_{\mathbf{e}, \mathbf{o}}$ & $\mathbf{A l}_{\mathbf{e}, \mathbf{o}}$ \\
\hline $\begin{array}{l}\text { Based on dissolved buffers along } \\
\text { transit stops in the study area }\end{array}$ & 5.77 & 11.09 & 5.85 & 6.43 & 3.04 & 32.18 \\
\hline Based on study area land use data & 7.29 & 14.84 & 11.40 & 10.14 & 5.33 & 48.99 \\
\hline \% not served & 20.75 & 25.24 & 48.68 & 36.65 & 43.00 & 34.32 \\
\hline
\end{tabular}

Analysis indicates that 48.38 percent of unemployed, 47.38 percent of population with auto-ownership 0 or 1, 58.97 percent of population between 15 to 64 years of age, and 40.32 percent of population with income less than $\$ 25,000$ in the entire study area are not being served by the transit system. The transit accessibility index based on demographic/socio-economic data for the entire study area by dissolving barriers for all transit stops $(376,550)$ is 55.31 percent of the of the maximum transit accessibility index based on demographic/socio-economic data at census block level $(842,620)$.

The results obtained also indicate that 20.75 percent, 25.24 percent, 48.68 percent, 36.65 percent and 43.00 percent of heavy commercial, light commercial, heavy industrial, light industrial, and institutional land use areas, respectively, are not being served currently in the study area. Overall, the transit accessibility index based on land use characteristics at the study area level is computed equal to 48.99. The same index for the entire study area based on dissolved barriers around transit stops is computed equal to 32.18. This indicates that 34.32 percent of the considered land use categories are not being served by the existing transit system in the study area. 
Figure 4 depicts transit accessibility indicators based on census block level data. Dark shaded census blocks are the ones with a high number of potential captive riders based on demographic/socio-economic data. Overlaying the existing transit route network clearly shows areas with greater number of potential captive riders that are not being served by the existing transit system. The figure provides valuable insights into extending existing routes or adding new routes to capture additional riders and increase transit market potential. Some examples for extension of an existing route and addition of a new route in Charlotte region are shown in Figure 4.

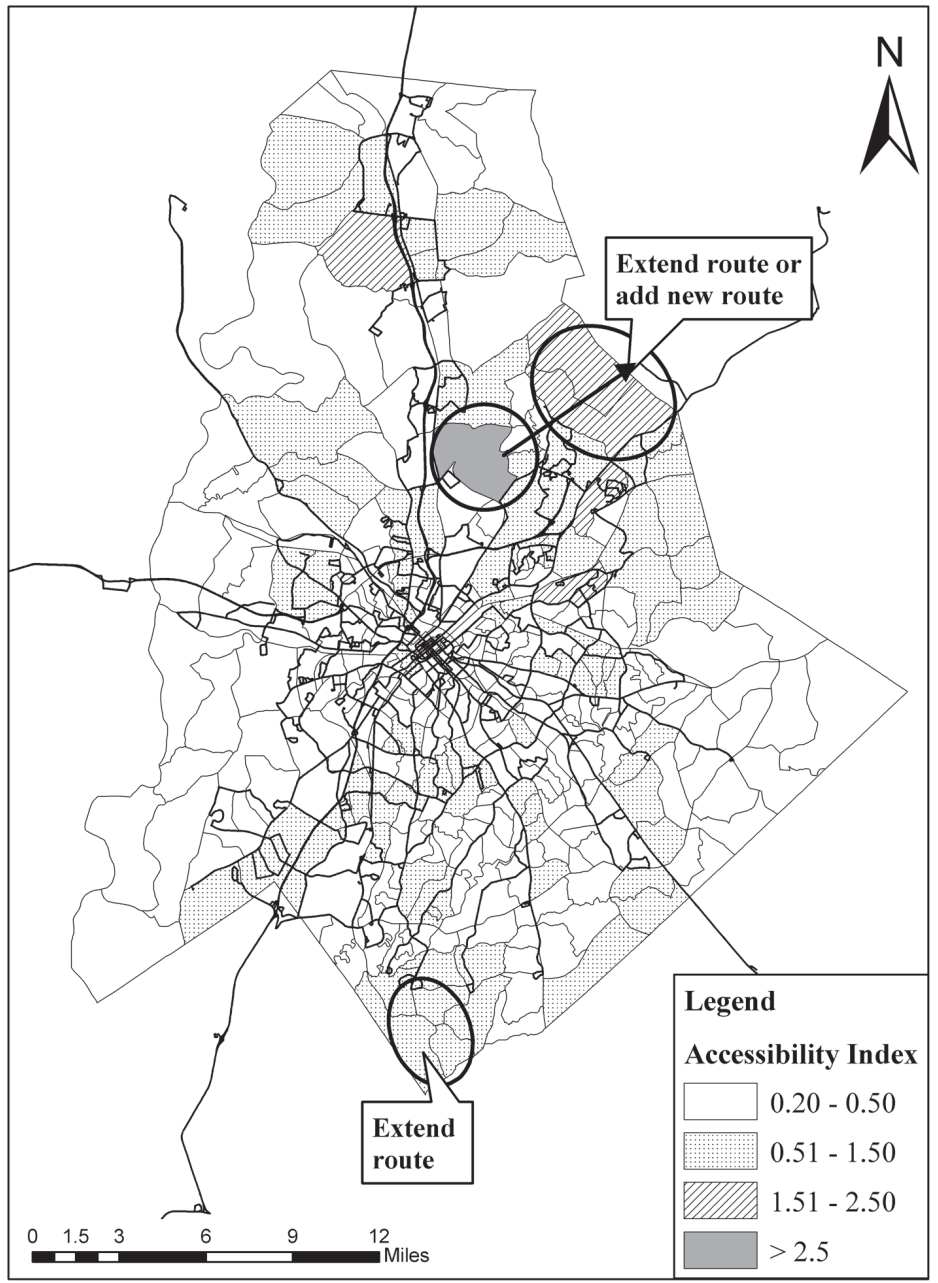

Figure 4. Map depicting accessibility index based on demographic/ socio-economic data and suggested extensions/new routes. 


\section{Statistical Analysis}

Statistical analysis (simple linear regression) was conducted to examine the relationship between 1 ) average daily boardings and the computed transit accessibility index based on demographic/socio-economic characteristics and land use characteristics for transit stops, and 2) average daily alightings and the computed transit accessibility index based on demographic/socio-economic characteristics and land use characteristics for transit stops.

Ridership data (boardings and alightings surveyed at transit stops) obtained from CATS were processed to estimate average daily boardings and lightings for each transit stop in the study area. The average daily boardings or alightings at a transit stop was considered as the dependent variable while the transit accessibility index based on demographic/socio-economic or land use characteristics was considered as the independent variable.

If a statistically significant relationship exists between the dependent and independent variables at a 95 percent confidence level (or level of significance lower than 0.05 ), then one can be fairly confident that the methodology developed can be used to compute the transit accessibility index and in the decision making process.

Statistical parameters (Table 4) obtained from linear regression analysis indicate that there is a statistically significant relationship between boarding or alightings and computed transit accessibility indices based on demographic/socio-economic and land use characteristics for transit stops. The T-Statistic is greater than 2, the P-value is less than 0.01 (99 percent confidence level), and the F-Statistic is greater than 4 (high) for all the tested scenarios shown in Table 4. Overall, results obtained show that there exists a statistically significant relationship between boardings or alightings and the transit accessibility index developed and used for analysis in this research.

The coefficient is positive, indicating that boardings or alightings increase as the transit accessibility index increases. In other words, one can say that having transit stops in locations with a high transit accessibility index based on criteria defined in this manuscript for a city similar in size and characteristics of Charlotte possibly increases market potential and ridership. Likewise, selecting routes with high transit accessibility indices based on criteria defined in this manuscript for a city similar in size and characteristics of Charlotte possibly increases market potential and ridership. 
Table 4. Statistical Analysis-Boardings or Alightings vs. Accessibility Index

\begin{tabular}{|l|c|c|c|c|c|}
\hline Category & Constant & Coefficient & T- Stat & P - Value & F - Stat \\
\hline \multicolumn{7}{|c|}{ Boardings vs. Transit Accessibility based on Demographic/Socio-economic } \\
\hline Transit stops & -10.70 & 39.37 & 14.16 & $<0.01$ & 200.56 \\
\hline \multicolumn{7}{|c|}{ Boardings vs. Transit Accessibility based on Land Use } \\
\hline Transit stops & -3.68 & 70.57 & 15.52 & $<0.01$ & 241.04 \\
\hline \multicolumn{7}{|c|}{ Alightings vs. Transit Accessibility based on Demographic/Socio-economic } \\
\hline Transit stops & -9.99 & 38.54 & 6.65 & $<0.01$ & 44.34 \\
\hline \multicolumn{7}{|c|}{ Alightings vs. Transit Accessibility based on Land Use } \\
\hline Transit stops & -3.71 & 70.99 & 7.47 & $<0.01$ & 55.88 \\
\hline
\end{tabular}

\section{Conclusions}

This manuscript presents a GIS-based methodology to compute an accessibility index for a transit stop and route and an entire study area. The methodology is illustrated using the city of Charlotte transit network that comprises 80 routes and 3,645 transit stops. The average transit accessibility index based on demographic/ socio-economic data for the transit stops in the study area is 0.72 , whereas the average transit accessibility index based on land use characteristics for the transit stops in the study area is 0.30 .

Analysis and assessment indicates that the transit accessibility index based on demographic/socio-economic data and land use data in the entire study area are 55.31 percent and 34.32 percent lower than the corresponding maximum potential transit accessibility index values, respectively. The computed transit accessibility indices were used to illustrate the identification of spatial gaps in transit accessibility so as to select new transit stop locations or relocate existing transit stops along a route. Illustrations also include area level analysis to identify new routes or to extend an existing route.

Statistical analysis conducted to assess the strength of the relationship indicates that a statistically significant relationship exists between boardings or alightings and accessibility index computed for analysis in this manuscript. This shows that adopting the proposed methodology to minimize spatial gaps and inaccessible areas increases transit ridership and market potential. 


\section{References}

Acs, G., and P. Loprest. 2005. Who are low-income working families? The Urban Institute, Washington, D.C. http://www.urban.org/UploadedPDF/311242_ working_families.pdf.

Ammons, D. N. 2001. Municipal Benchmarks: Assessing Local Performance and Establishing Community Standards, 2nd Edition. Sage Publications, Inc., Thousand Oaks, California.

Beimborn, E. A., M. J. Greenwald., and J. Xia. 2003. Accessibility, connectivity and captivity impacts on transit choice. Center for Urban Transportation Studies, University of Wisconsin, Milwaukee, Wisconsin.

Bhat, C., S. Handy, K. Kockelman, H. Mahmassani, L. Weston, A. Gopal, and I. Srour. 2002. Development of urban accessibility index: A summary. Center for Transportation Research, The University of Texas at Austin, Austin, Texas.

Kimpel, T. J., K. J. Ducker, and A. M. El- Geneidy. 2007. Using GIS to measure the effect of overlapping service areas on passenger boarding at bus stops. Urban and Regional Information Systems Association Journal. http://urisa.org/kimpel.

Kuby, M., C. Upchurch, M. Zoldak, and A. Barranda. 2004. Using GIS to generate mutually exclusive service areas linking travel on and off a network. Journal of Transport Geography 12: 23-33.

Lee, B. H. Y. 2004. Parcel-level measure of public transit accessibility to destinations. TransNow Student Conference, Portland State University, Portland Oregon.

Murray, A. T. 2003. A coverage model for improving public transit system accessibility and expanding access. Annals of Operations Research 123: 143-156.

Pulugurtha, S. S., S. S. Nambisan, and N. Srinivasan. 1999. Evaluating market transit potential and selecting locations of transit service facilities using GIS. Journal of Public Transportation 2(4): 75-94.

Pulugurtha, S. S., V. R. Duddu, and R. Mora. 2011. Identifying Spatial Gaps in Transit Accessibility to Maximize Market Potential. Proceedings of the First T\&DI Congress 2011, Chicago, Illinois.

Saka, A. A. 2001. Model for determining optimum bus-stop spacing in urban areas. Journal of Transportation Engineering 127: 195-199. 
Sanchez, T. W. 1998. the connection between public transit and employment. Center for Urban Studies, School of Urban Studies and Planning, Portland State University, Portland Oregon.

\section{About the Authors}

SRINivas S. Pulugurtha (sspulugurtha@uncc.edu) is currently working as an Associate Professor of Civil and Environmental Engineering and an Assistant Director of the Center for Transportation Policy Studies at the University of North Carolina at Charlotte. His area of interests includes traffic safety, GIS applications, transportation planning/modeling, traffic simulation, and development of decision support tools.

VenKatA R. DuDDu (vduddu@uncc.edu) is currently a Ph.D. student in Transportation Engineering at the University of North Carolina at Charlotte. He has an M.S. in Civil Engineering from the University of North Carolina at Charlotte.

RAKESH MORA (rmora@uncc.edu) is an M.S. student in Transportation Engineering at the University of North Carolina at Charlotte. 